Hotchis, J. E. (1951). J. gen. Microbiol. 5, 609-618.

\title{
The Influence of Acridines on the Interaction of Staphylococcus aureus and Staphylococcus $K$ phage
}

\author{
By J. E. HOTCHIN \\ National Institute for Medical Research, Mill Hill, London, N.W.7
}

SUMMARY: The inhibition of multiplication of staphylococcus $K$ bacteriophage by six acridines and two amidines has been studied using mass lysis and one-step growth curve techniques. The effect of the different inhibitors upon the growth rate of the organisms was found to vary from below 5 to $100 \%$ inhibition at a concentration sufficient to double lysis-time. Results of one-step growth curves showed a progressive decrease in burst size with increasing concentrations of inhibitor, and gave no evidence for any variation in sensitivity to inhibitor during the latent period. Using higher concentrations of inhibitor a zone was found where accelerated lysis occurred with loss of phage.

The bacteriophages are being studied increasingly in experimental systems for the investigation of the factors determining the interaction between virus and host cell. The aim of the work described here was to ascertain the extent to which the lysis of a pure culture of Staphylococcus aureus by a given staphylococcal phage is affected by acridines, certain of which have been reported (Fitzgerald \& Babbitt, 1946) to inhibit lysis of an Escherichia coli phage system. When an inhibition of mass lysis had been demonstrated in the Staph. aureus phage system, more detailed studies were made using one-step growth curves. An attempt was made to throw some light upon the processes of bacteriophage multiplication from observations of the way in which the inhibitors acted.

\section{MATERIALS AND METHODS}

Organisms. All experiments were performed on the Oxford ' $H$ ' strain of Staph. aureus (N.C.T.C. No. 6571A), grown on slopes of $2 \%$ agar in tryptic digest broth (Hartley, 1922), and subcultured daily.

Bacteriophage. The anti-staphylococcus phage staphylococcus $K$ originally obtained from Dr A. P. Krueger was used throughout.

Inhibitors. Five acridine compounds, two compounds with similar structure (rhodamine and fluorescein) and two with similar action but different structure (propamidine and pentamidine), were tested (see Table 1). Concentrations are expressed throughout in g.mol./l. (M) and micromols/l. $(\mu \mathrm{M})$, for convenient comparison. Solutions $(0.01 \mathrm{M})$ were made up in distilled water and sterilized by filtration through $0 \cdot 7 \mu$. Gradocol membranes.

Bacterial growth. Two methods of estimation were used: the plate count of colonies of viable organisms, and measurement of the turbidities of cultures using a Hilger 'Biochem' absorptiometer.

Bacteriophage growth. Phage suspensions were titrated by plaque-counts on $1 \%$ agar plates. 
One-step growth curves. The technique used was that devised by Delbrück \& Luria (1942) with modifications to permit the application and removal by dilution of inhibitor for varying periods during the latent period, and to permit several experiments to be run concurrently. The latter was achieved by staggering the sampling times by half-minute intervals. A final $10^{-6}$ dilution of the experimental tube permitted samples to be plated directly and gave a convenient count up to 200 plaques. The action of the inhibitor was arrested by a $10^{-2}$ dilution. Initial concentrations of viable organisms and phage in the experimental system were: organisms $3 \times 10^{7} / \mathrm{ml}$; ; bacteriophage $4 \times 10^{7} / \mathrm{ml}$. Adsorption for $5 \mathrm{~min}$. gave in control tubes an infective ratio of less than 1 and a burst size of about 60 . Samples of the same batch of broth were used throughout to ensure uniformity of the nutrient medium; experience showed this to be most important.

Bacterial lysis. Degrees of mass lysis were measured photoelectrically. Turbidimetric readings were usually taken over a.period of $c .6 \mathrm{hr}$. and then plotted.

Influence of inhibitor on lysis. Organisms in the logarithmic phase of growth in broth were spun down and resuspended in fresh broth to provide a suspension of standard turbidity. Five concentrations of inhibitor were used in duplicate for each experiment, with two control tubes, one for organism growth and one for lysis by phage. To half the tubes sufficient phage was added in $\mathbf{0 . 1} \mathrm{ml}$. broth to give complete lysis in the control tube in 2 to $4 \mathrm{hr}$. The other six tubes received no phage, to provide curves for the direct effect of inhibitor upon bacterial growth. Immediately after addition of phage, all tubes received $2 \mathrm{ml}$. of the standard suspension of organisms, and were incubated at $37^{\circ}$. The initial viable count of bacteria in these experiments was $1.5 \times 10^{8}$ organisms $/ \mathrm{ml}$, and the initial phage titre was $1 \times 10^{8}$ particles $/ \mathrm{ml}$.

\section{RESULTS}

\section{The effect of acridines on staphylococci}

All inhibitors were first tested for their power to prevent completely the growth of a small inoculum of Staph. aureus in broth during 24-hr. incubation. The minimal bacteriostatic concentration was taken as a guide for the concentrations to be used later with the phage +host-cell systems. Graded effects upon the rate of growth of the organisms were obtained in all cases with concentrations of inhibitor below the level needed to prevent growth. The results of these experiments are summarized with those of lysis experiments, in Table 2, cols. 3 and 4.

\section{The action of acridines on staphylococcus $\boldsymbol{K}$ bacteriophage}

The effects of most of the inhibitors were tested upon staphylococcus $K$ phage alone, at $37^{\circ}$ in broth. Previous results obtained by Fitzgerald \& Babbitt (1946) and by Foster (1948) showed the direct inactivation of coli $T$ phages by acridines to be negligible at concentrations which inhibited the growth of phage in the presence of its host organism. This was confirmed 
Table 1. Structural formulae of compounds tested<smiles>NC1CCC(C2=NC3CCNCC3CCC2)CC1</smiles>

$\mathrm{HNO}_{3}$<smiles>NC1CCC2CC3CCCNC3NC2C1</smiles><smiles>NC1C2CCCCC2NC2CCCCC21</smiles>

$\mathrm{HCl}$<smiles>CCN(CC)CCCCNC1C2CC(OC)CCC2=NC2CC(Cl)CCC21</smiles><smiles>CCOCC1CCC2N=C3CN(N)CCC3CC2C1N</smiles>

$\mathrm{HCl}$

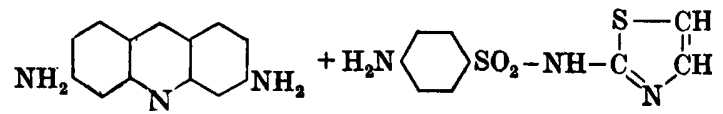

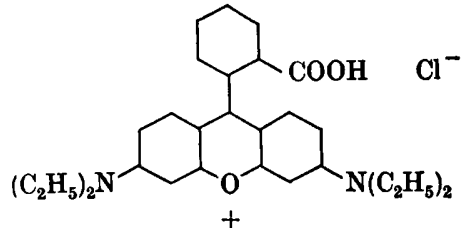<smiles>COC(=O)C1CCCCC1C1CC2COCC[C@H]2OC2CCCCC21</smiles><smiles>N=C1CCC(OCCCOC2CCC(N)(N)CC2)CC1</smiles>

As above $n=5$

Mepacrine (HK)

Rivanol (HK)

Flavazole (Boots)

Rhodamine B (BDH)

Fluorescein (Harr.)

Propamidine isothionate ( $M \& B$ )

Pentamidine isothionate (M \& B)

Sources of compounds used: (ICI)=Imperial Chemical Industries Ltd.; (Boots)=Boots Pure Drug Co.; (HK)=Dr H. King; (BDH) =British Drug Houses Ltd.; (Harr.)=Harrington Bros.; (M \& B) = May \& Baker. 
Table 2. Critical concentrations and $p H$ values of inhibitors affecting Staph. aureus and staphylococcus $K$ phage in broth medium $\mathrm{pH}$ 7-7·4

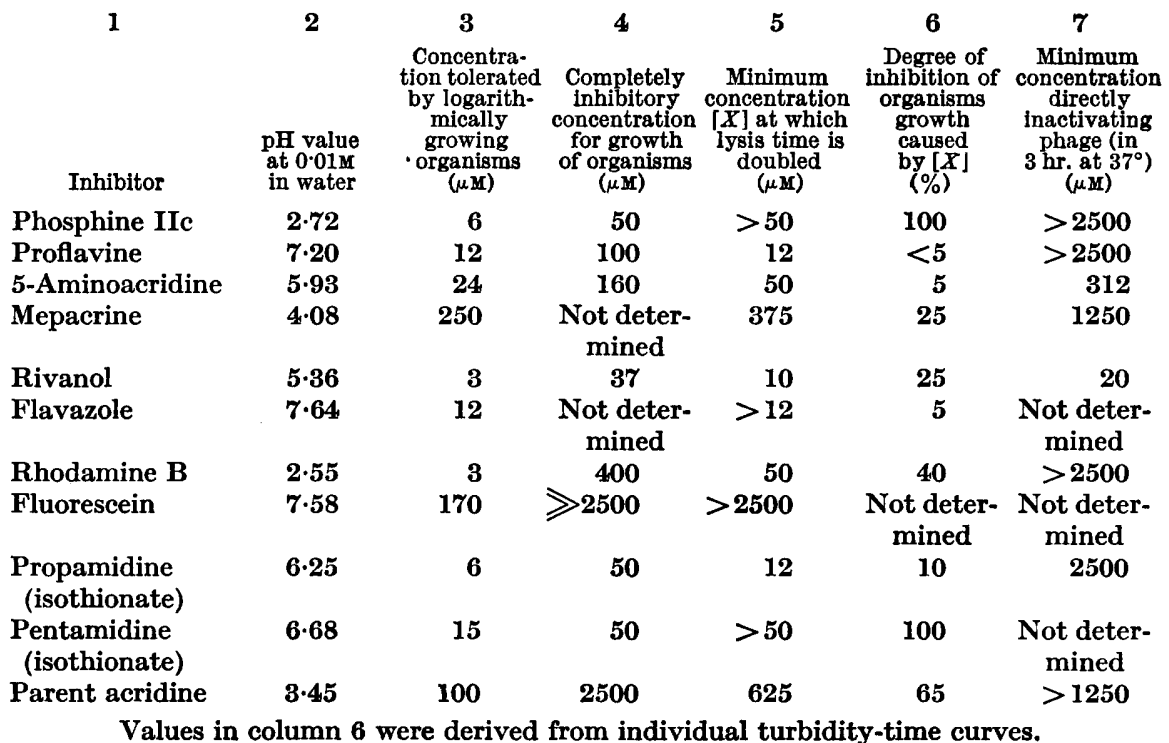

using $T_{2}$ phage, which was not affected by $1000 \mu \mathrm{M}$. acridine at $37^{\circ}$ in broth for $20 \mathrm{hr}$. Staphylococcus $K$ phage, however, was more susceptible to inactivation by acridines than was $T_{2}$. The relative inactivating concentrations of most of the inhibitors studied are shown in Table 2, col. 7 .

\section{The effect of acridines on the lysis of staphylococci by phage}

Turbidimetric results. A typical series of turbidity-time curves obtained in experiments with 5-aminoacridine(Figs. 1 and 2) illustrates the effect of various concentrations of this compound on the growth of staphylococci and their lysis by phage. Similar curves were determined for each of the substances tested. The results are summarized in Table 2, cols. 5 and 6. It is interesting to compare the figures for maximum concentration of inhibitor tolerated by the organism without noticeably (within $5 \%$ ) altering its logarithmic growth rate (column 3 ), and the minimum concentration $[X]$ of inhibitor required to double the lysis time (column 5). It is evident that inhibition of lysis and of the growth of the organism are not always parallel. A strong inhibitory effect on lysis is almost always associated with some interference with the metabolic processes of the host as shown by a measurable decrease in growth rate, the degree of interference varying with the compound. Proflavine, for example, inhibited lysis significantly at a concentration where only a very slight inhibition of host multiplication was detectable.

The effects of increasing the concentration of inhibitor substance on the shape of the curves for growth and lysis were studied, and some interesting 


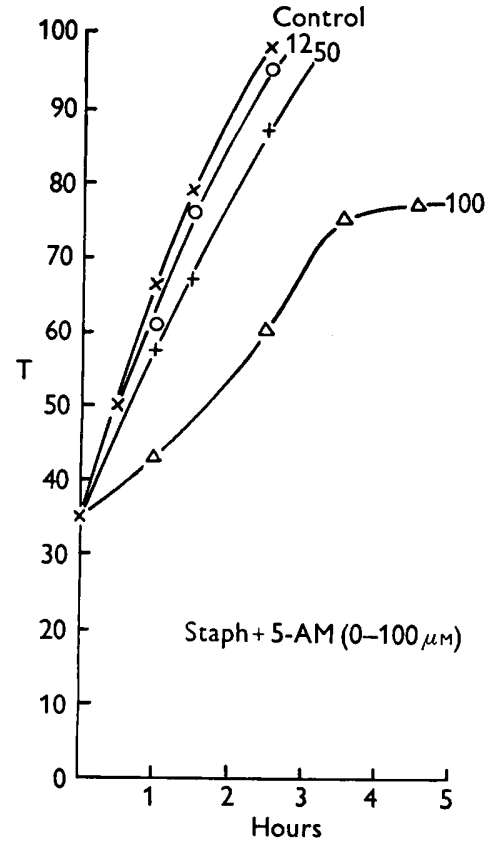

Fig. 1

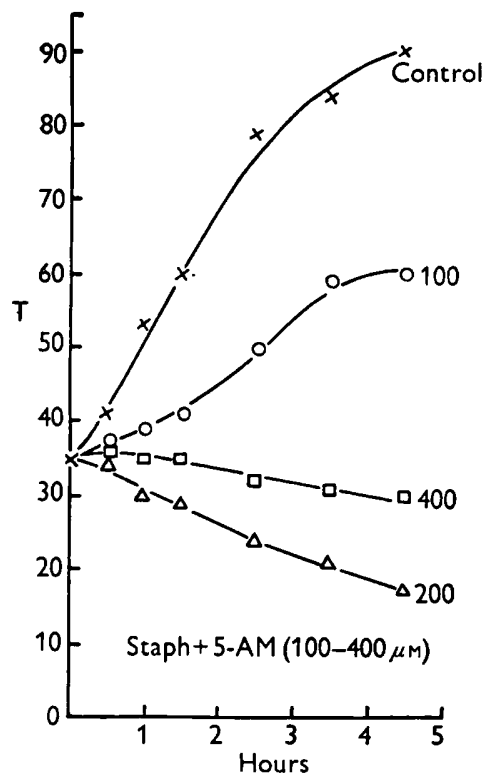

Fig. 3

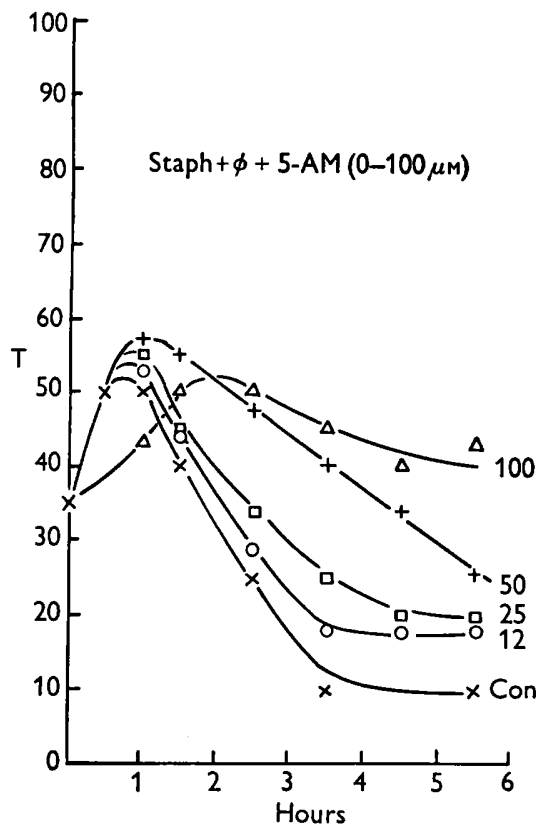

Fig. 2

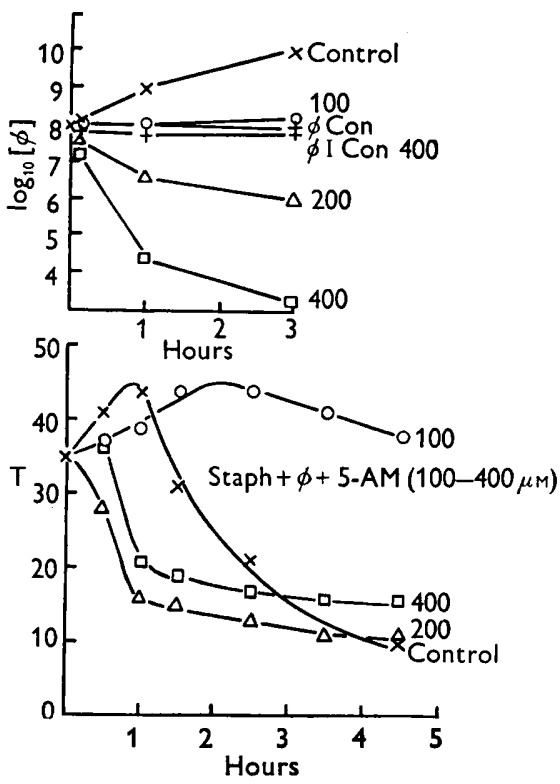

Fig. 4

Figs. 1-4. Effects of 5-aminoacridine upon turbidity-time curves of Staph. aureus 'Oxford' cultures with and without phage. Figures show concentrations of acridine in $\mu \mathrm{M}$.

For abbreviations see p. 618 . 
new phenomena observed. Figs. 3 and 4 show the curves obtained in such an experiment, using 5-aminoacridine. The concentration of $100 \mu \mathrm{M}$. caused long inhibition of lysis, but the curve obtained with a twofold increase of inhibitor to $200 \mu \mathrm{M}$. was significantly different. Here, lysis was greatly accelerated, beginning at once and in $1 \mathrm{hr}$. reaching a degree which was only reached in $3 \mathrm{hr}$. by the control. The $400 \mu \mathrm{M}$. tube showed a similar curve but the effect was less well-marked, with a shift back towards the control. The accompanying changes in titres of available phage are shown in the upper part of Fig. 4.

The growth of the organisms alone was affected in a părallel manner (Fig. 3): $100 \mu \mathrm{M}$. inhibitor gave approximately $50 \%$ inhibition of growth. With $200 \mu \mathrm{M}$. however there was no growth, but the turbidity of the culture steadily diminished. Increasing the concentration of 5-aminoacridine still further, to $400 \mu \mathrm{M}$., produced a similar but smaller effect.

Appreciable multiplication of phage occurred in the control lysis tube, and, to a lesser extent, in the presence of $100 \mu \mathrm{m}$. inhibitor. At concentrations 200 and $400 \mu \mathrm{M}$., the titre of phage fell off steeply. In the absence of staphylococci these concentrations of inhibitor produced no comparable change in the titre of phage over the same period of incubation. The direct effect of $312 \mu \mathrm{m}$. (see Table 2, col. 7) only accounts for a small fraction of the inactivation. Apparently, therefore, phage at low concentration can lyse organisms which have their growth processes entirely inhibited, but the phage itself does not multiply under these conditions. This accelerated lysis and decrease in turbidity of organisms was also observed with propamidine.

\section{Results using one-step growth curves}

The curves in Fig. 5 show the effects of different concentrations of 5-aminoacridine upon the one-step growth curve of staphylococcus $K$ phage. The system was exposed to the inhibitor from the fifth minute onwards. A progressive increase in concentration of inhibitor caused a progressive decrease in burst size, until at $\mathbf{1 0 0} \mu \mathrm{M}$. no increase in phage was detectable. This level corresponds to the 'completely inhibitory' concentration of Foster (1948). In an analysis of phage infection, its initial adsorption by the organism may be studied, but from there onwards until new phage is liberated during lysis, what happens within the cell is largely unknown. It was thought that an investigation of the effect of allowing the inhibitor to act for a given time at different stages within the latent period might prove illuminating and perhaps indicate differences in host-sensibility at different phases of phage multiplication. The result of the first experiment of this kind is shown in Fig. 6. A concentration of $50 \mu \mathrm{M}$. 5-aminoacridine was added at progressively later stages in the latent period. The burst size decreased in value proportionately to the time of contact with the drug. The curves indicate that the latent period was unaffected, and this was confirmed in other experiments.

The effect of time of exposure of the multiplying phage to $50 \mu \mathrm{M}$. inhibitor was investigated further by arresting the action of the drug after varying periods within the latent period. This was achieved by finally diluting the 
inhibitor one hundredfold. The inhibitor decreased the burst size and extended the latent period proportionately to the duration of its action. The effect on burst size was less than when the inhibitor was added and thereafter allowed to operate continuously during the latent period. It must be remembered in the latter case that besides acting in the latent period, the inhibitor continued to act during the rise period, which in the inhibited system was sometimes twice as long as the usual $10 \mathrm{~min}$. The shorter exposure of the system, when inhibition was removed, allowed its recovery from the effects of the inhibition. The amount of recovery possible appeared to be directly proportional to the

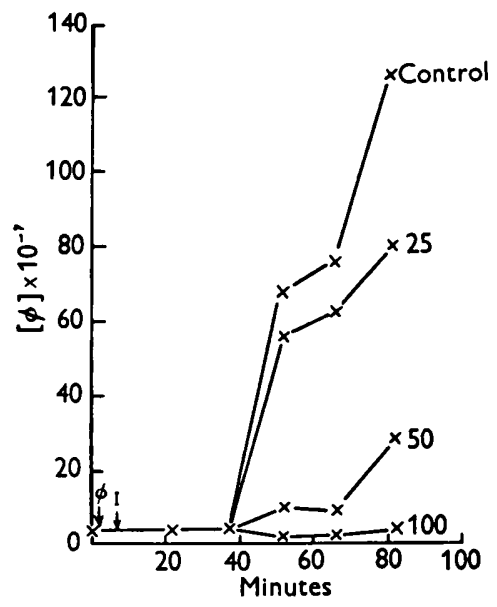

Fig. 5

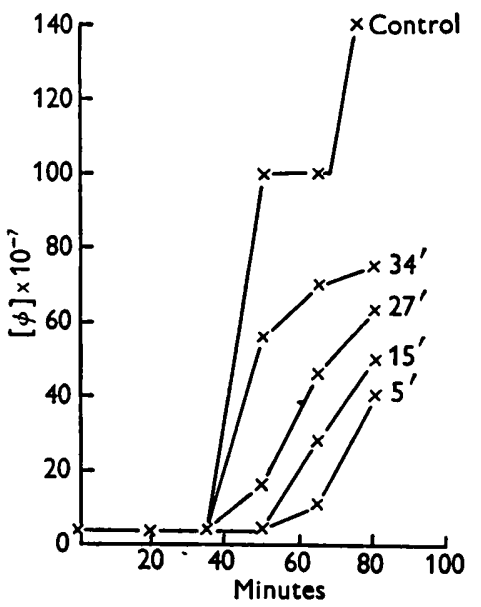

Fig. 6

Effects of 5-aminoacridine upon one-step growth curves of staph. $K$ phage.

Fig. 5. Figures show concentration of 5-aminoacridine in $\mu \mathrm{M}$.

$\phi=$ Time of infection of Staph. aureus 'Oxford' with $\phi$.

$I=$ Time of addition of inhibitor.

Fig. 6. Figures show times of addition of 5-aminoacridine to give $50 \mu \mathrm{M}$. concentration. Infection with $\phi$ was at zero time.

period remaining before lysis was due to be complete, i.e. the end of the rise period.

When the system was exposed to inhibitor for 10-min. periods, during each of three consecutive thirds of the latent period, no differences in the amount of inhibition produced could be detected.

A similar comparison was made of the effects of the sub-inhibitory concentration $50 \mu \mathrm{M}$. with those of the completely inhibitory concentration of $200 \mu \mathrm{M}$. In each case the inhibitor was allowed to act for a 10-min. period in the middle of the latent period (Fig. 7). The capacity of the system to recover is well illustrated. In tube $B$ where the concentration was $50 \mu \mathrm{M}$. recovery is almost complete. At the concentration of $200 \mu \mathrm{M}$., however, the burst size is decreased and the latent period extended by an interval equal to that of the exposure period. Reference to Fig. 1 shows that at this concentration the 
multiplication of organisms is completely inhibited. Also at this concentration accelerated lysis is at a maximum (see Fig. 4) and is invariably accompanied by a decrease in phage titre.

The effect of nucleic acids on the inhibition by acridines of the lytic action of phage and the growth of organisms

The observation of Fitzgerald \& Lee (1946), of Smith (1949) and of Foster (1948) that yeast ribonucleic acid counteracts the effects of acridines upon lysis by phage was confirmed for Staph. aureus and staphylococcus $K$ phage.

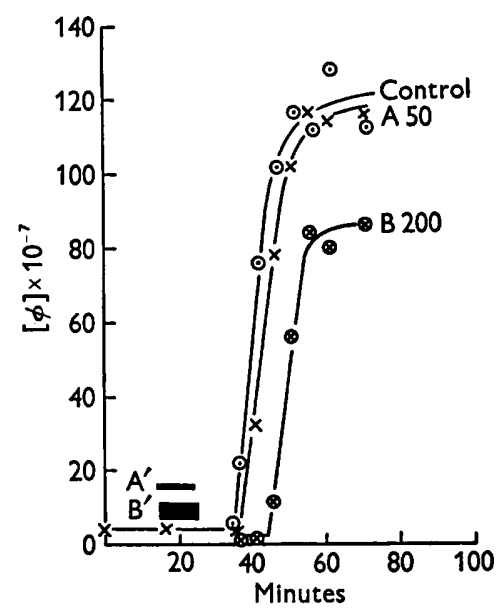

Fig. 7. The effect of $50 \mu \mathrm{M}$. 5-aminoacridine $(A)$ and $200 \mu \mathrm{M}$. 5-aminoacridine $(B)$. Exposure time to inhibitor during the latent period is represented as $A^{\prime}$ and $B^{\prime}$.

Commercial preparations of nucleic acid were added before the acridine. Ribonucleic acid (RNA) was inferior to thymus deoxyribonucleic acid (DNA) in counteracting the effect of phosphine IIc and 5-aminoacridine. When the w/v concentration of DNA was 25 times that of the acridine, the inhibition of lysis by 5 -aminoacridine was completely. antagonized, whereas RNA did not antagonize unless its concentration was 100 times that of the acridine. Reversal of the inhibition of growth of organisms by acridines was obtained by adding the nucleic acid after an interval but recovery only occurred gradually after a long lag and for this reason was not demonstrable with a phage-inhibitor-organism system.

\section{DISCUSSION}

It is essential, when studying whether or not selected compounds inhibit or modify the interaction of viruses with their host cells, to distinguish between the direct effects of the compound on the host and on the outcome of the infection, so that the therapeutic value of that compound against virus infection may be assessed. The compounds studied here were without effect 
on the free phage and on its adsorption by the cell at concentrations completely inhibitory for the organisms; the inhibition observed therefore occurred at some point in the reactions involving virus synthesis or release.

It was found that with a compound active against the phage a concentration of this compound below that which is detrimental to the growth of the host organism causes a decrease in the average phage multiplication/cell, as revealed by a fall in burst size. As the concentration is raised further the burst size progressively diminishes with little, if any, change in the latent period of infection. With the inhibitor at a concentration where definite inhibition of the growth of the host organism occurs, the latent period of infection is prolonged and phage multiplication ceases, as indicated by zero burst size. The one-step growth curve then suggests an indefinitely prolonged latent period. When, however, such an inhibited system is diluted into broth containing no inhibitor, then lysis will occur with a high burst size. It appears that when the growth processes of the host cell are arrested by inhibitor, that compound thereafter renders the cell more susceptible to lysis either through the agency of phage itself or by the combined effects of phage and autolytic enzymes, so that rapid lysis occurs with loss of infecting phage.

These observations on the inhibition of the interaction of phage and host staphylococci by acridines are in keeping with those of Fitzgerald \& Babbitt (1946) and of Foster (1948), carried out with Esch. coli and the $T$ series of phages. Their experiments were made in chemically defined medium; the present ones were all performed in broth, which gives a well-defined latent period and a much sharper rise period, so that a more clear-cut picture of the inhibitory effect in one-step growth curves was obtained. Smith (1949) reported results with phosphine GRN and acriflavine on several organisms, including two strains of Staph. aureus, and a polyvalent staphylococcus phage preparation. The concentrations he found to be inhibitory to phage multiplication are similar to those used here for the same or closely allied acridines. I did not observe the stimulation of lysis by phosphine at low concentrations reported by Smith with phosphine GRN, but phosphine IIc caused accelerated lysis at higher concentrations.

The fact that no variation in sensitivity was found during the latent period suggests either that phage multiplication proceeds by single fission continuously susceptible to acridine inhibition, or that the normal continuous production of some essential intermediate or precursor substance by the host organism is progressively retarded by the inhibitor with a consequent decrease in phage multiplication, as evidenced by the one-step growth curves. The latter process is considered to be the more likely explanation of the mode of action of the acridines, from the results of these experiments. But it must be remembered that the technique used measures only mature 'viable' phage particles; any phage particles formed during inhibition that were altered so as to render them non-viable would give no indication of their presence.

It is believed that the phenomenon here called accelerated lysis has not previously been described in connexion with high concentrations of acridines or propamidine. It resembles the phenomenon described by Cohen (1949) in 
which $E$ sch. coli was rapidly lysed without phage increase when its respiration was prevented by nitrogen or cyanide. Elford (1948) recorded the accelerated lysis of Staph. aureus in the presence of penicillin; this occurred with the production of active phage which had been able to multiply, thus differing from the accelerated lysis due to acridines whereby phage is lost. The numbers of phage particles in the above experiments were far too low for the effect to be due to 'lysis from without' as described by Delbrück (1940).

The close similarity of the action of the acridines and propamidine as phage inhibitors is interesting as it is in accordance with the work of Gotts \& Sevag (1948) who showed very close parallelism between the effects of these two drugs on the dehydrogenase inhibition of pneumococci.

It is a pleasure to thank Dr W. J. Elford for his invaluable advice and criticism in the course of this work.

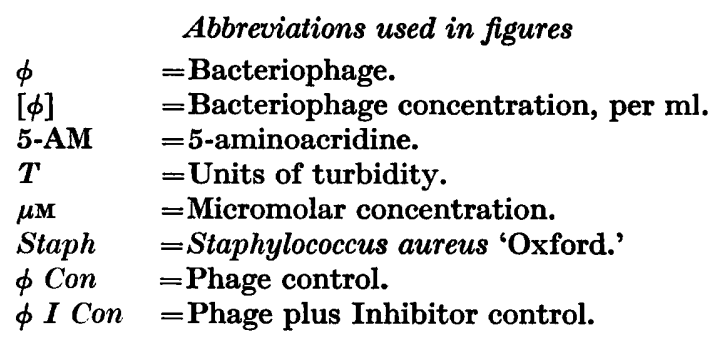

\section{REFERENCES}

CoHen, S. S. (1949). Growth requirements of bacterial viruses. Bact. Rev. 13, 1.

Dembrück, M. (1940). The growth of bacteriophage and lysis of the host. J. gen. Physiol. 23, 643.

DelBRÜCK, M. \& LưrA, S. E. (1942). Interference between bacterial viruses. I. Interference between two bacterial viruses acting upon the same host, and the mechanism of virus growth. Arch. Biochem. 1, 111 .

Elford, W. J. (1948). The influence of antibacterial substances on the interaction of bacteria and bacteriophages. I. The influence of penicillin. J.gen. Microbiol. $2,205$.

Fitzgerald, R. J. \& BabbitT, D. (1946). Studies on bacterial viruses. I. The effect of certain compounds on the lysis of Escherichia coli by bacteriophage. J. Immunol. 52, 121.

Fitzgerald, R. J. \& LEe, M. E. (1946). Studies on bacterial viruses. II. Observations on the mode of action of acridines in inhibiting lysis of virus-infected bacteria. J. Immunol. 52, 127.

Foster, R. A. C. (1948). An analysis of the action of proflavine on bacteriophage growth. J. Bact. 56, 795.

Gotrs, J. S. \& SevaG, M. G. (1948). Enzymatic studies on the mechanism of the resistance of pneumococcus to drugs. I. Studies of the dehydrogenase activities and inter-relationships of pneumococci susceptible and resistant to acriflavine, atebrine, optochin, propamidine and sulfonamides. J. Bact. 56, 709.

Hartley, P. (1922). The value of Douglas's medium for the production of diphtheria toxin. J. Path. Bact. 25, 479.

Smrth, A. G. (1949). The effect of acriflavine and phosphine GRN on lysis of bacteria by bacteriophage. J. Immunol. 61, 57 .

(Received 11 July 1950) 